\title{
Performance Measurement Model of Mobile User Connectivity in Femtocell/Macrocell Networks using Fractional Frequency Re-use Scheme
}

\author{
Mehrin Anannya \\ Information \& Communication Technology \\ Bangladesh University of Professionals \\ Mirpur Cantonment, Mirpur-12, Dhaka, Bangladesh
}

\author{
Riad Mashrub Shourov \\ Skills for Employment Investment Program (SEIP) \\ Prabashi Kallyan Bhaban, Ramna, \\ Dhaka, Bangladesh
}

\begin{abstract}
Technologies are traversing to its new dimensions every day. As part of this progression, mobile cellular system is at the summit of its constant advancement. The usage of Femtocells in mobile cellular system has created a massive impact on its architecture. Likewise, the incorporation of femtocells in macrocells for 4G mobile network communication services (like-voice calls, data services, etc.) among mobile stations within few meters has been one of the promising approaches. There is a femto access point (FAP) in Femtocell which handles the authorization of the user around it. Among the three various access methods, FAP allows only the authorized users except the macro cell users in Closed Access Method (CAM). But for Open Access Method (OAM), any type of crossing macrocell user within the radio coverage of femtocell and the femtocell users can get FAP access. To reduce the crosstier interferences OAM is more efficient, because it deals with both type of users within the femtocell coverage. This paper proposes a performance measurement model for mobile connection probability depending on the mobility factor of mobile users and the communication range in femtocell/ macrocell networks. Furthermore, a derivation has been done to get the optimum result from the outage and connectivity probability under different number of femtocells and mobile users. Finally, to maximum the spectral efficiency for the probable frequency allocation, a Fractional Frequency Re-use scheme among the networks has been proposed.
\end{abstract}

Keywords-Femtocell; macrocell; cross-tier interferences; cotier interferences; closed access methods; open access methods; connectivity probability; mobility factor; outage probability; fractional frequency re-use scheme

\section{INTRODUCTION}

With the advancement of technology, wireless mobile communication is on high demand around the world. This demand arises not only for the voice communication but also for data services. The need for consistent connectivity between the users at two ends providing high speed communication with low cost and without any loss of data and interruption are increasing. So, a high-speed voice and data transmission such as, voice calls, video calls and rapid and faster internet facilities having clear video images without any kind of interruption over the network and without any loss, needs to be ensured to satisfy consumers by enhancing the system coverage and capacity and reducing the capital. Depending on the different operators, the mobile cells communicate with their respective base stations. In this case, the base stations are considered as the Macrocell Access Point (MAP), which provides an area of coverage to the mobile users. A Macrocell is nothing but a cell that provides wide radio coverage with high power cellular base station in a mobile phone network (tower). Due to substantial wireless communication between the mobile users, its workload climbs along with the increase of mobile users.

\section{A. Necessity of Femtocell Considering the Problems of Macrocell in Increasing the Overall Performance and Signal Quality of Mobile Communication}

To serve this vast number of mobile users, the performance and quality of the macrocell degrades due to slow connectivity, call drops, reduction of sound intensity, etc. Owing to this, co-tier and cross-tier interference also increases.

This workload of macrocells can be subdivided among some femtocells which work within the common home range. A femtocell is a short-range home area broadband network which gives coverage of about 10 meters providing better indoor voice and data communication. A femtocell has a base station named Femtocell Access Point, which mainly communicates with the Macrocell to build its own network under that Macrocell. Since, in this kind of network transmitter and receiver remain very close to each other, a high-quality link is created between them providing large number of spatial reuse with low power transmission and power wastage. It is seen that, $2 / 3$ of voice communication and $90 \%$ of data communication occur within the home range of a mobile user. So, for enterprise or home environments, to offload the traffic on the macrocell by using increasing system capacity, providing high probability of connectivity and utilizing spatial reuse of resources which provides highest spectrum efficiency, so that every user located at every corner of the macrocell can have clear connectivity, femtocell needs to be used. Due to femtocell, the cross-tier interference and handoffs under the macrocell decreases.

The users can access femtocells depending on three access mechanisms, namely, Closed Access Mechanism (CAM), Open Access Mechanism (OAM) and Hybrid Access Mechanism (HAM). In this research, we will utilize open access mechanism for Femtocells. 
Macrocell and femtocell has been shown in Fig. 1 and 2, respectively.

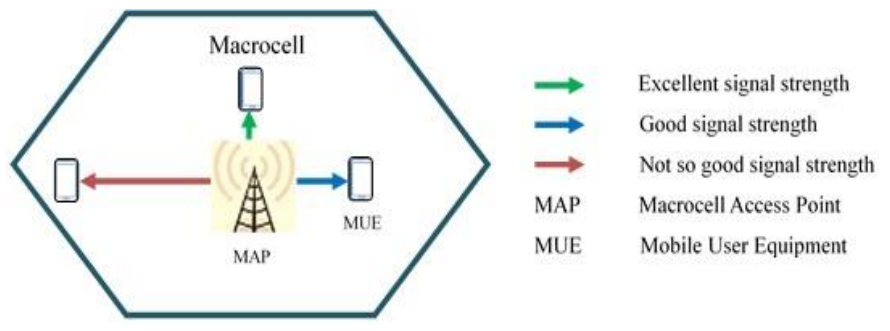

Fig. 1. Macrocell.

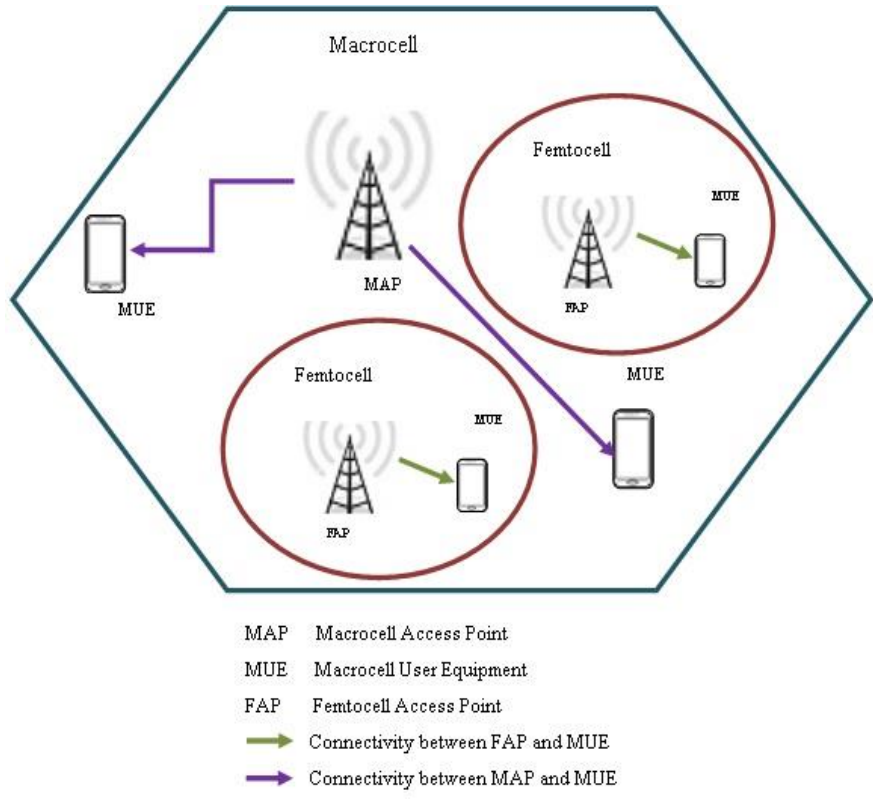

Fig. 2. Femtocell.

\section{B. Objectives}

- Observe the disadvantages of macrocells and focus on the advantages of femtocells based on the co-tier and the cross-tier interference.

- Develop a performance measurement model in order to observe the variation of connectivity probability against the number of mobile users in Open Access Method environment.

- Derive the outage probability in terms of mobile users and threshold of detection (SINR).

- Evaluate the variation of spectral efficiency against SINR.

- Finally, introduce a proposal of a fractional frequency re-use scheme for Self-Organization Network (SON) based on femtocell architecture.

\section{Scope}

To increment the connectivity probability by reducing outage probability beyond the level that has been proposed.

\section{LITERATURE REVIEW}

\section{A. Background}

Firstly, the connectivity with the femtocell needs to be confined which is maintained by access control of the femto cell. Regarding this, the merits and demerits of existing access methods of femtocells emphasizing on its technical impact and also a business model is described in [1] which headed towards the necessity of hybrid access methods along with several other models. In [2] a stochastic geometric model has been employed to enhance the spatial reuse of the femtocells meeting the per-tier outage constraint using cognitive radio. At the Primary User (PU) transmitter, a capacity-outage probability to the PU better than the beacon transmitter has been examined in [3] with the statistical model that has been developed so far which imposes less interference. Furthermore, the model is prolonged in investigating the cooperative sensing effect and capacity-outage performance for maximizing the likelihood cooperative detection techniques. A brief idea about the requirement of the femtocells along with the technical and business arguments for femtocells and its challenges, way of overcoming the challenges and the state-of-the-art on each front of it has been discussed in [4]. Not only for wireless communication but also in case of wired communication, it is seen that the performance of femtocells is better than the macrocells. The simulation result in [5] shows that via vehement wireless spectrum spatial reuse, the sententious capacity gain of the areal (per single area) can be achieved giving a strong signal strength in case of femtocell and weak signal strength in macrocell. In [6] an interpretive model framework has been demonstrated for computing the plausibility of mobile user connectivity depending on the femtocell density, communication range, mobility factor, and user density in femtocell/ microcell, which by examining the performance of outage probability and spectral efficiency in that networks found to be efficient during planning of Macro cellular networks integrated with Femto cellular networks. But the consummation of the transmission quality largely bets on the interference for different distances. For different measurements of distance, the interference aware and SINR estimation of femtocell networks by using frequency reuse mechanisms for LTE has been done in [7,1]. In [8], an algorithm on sub-channel allocation was developed using graph-theoretic approach in which a grouping of femtocell users is made into disparate clusters for subduing the interference within them and optimizing the femtocell throughput in densely populated femtocells deployment with adaptive power allocation to enhance the system throughput. In [9] a discussion has been done about a survey on different level of development approaches, qualitative comparison and open challenges of interference along with asset management in orthogonal frequency-division multiple access (OFDMA) based femtocell networks. In [10] discussions have been made on evolution, characteristics, design and deployment aspects for femtocell discovery by active call hand-in and idle mobiles switching from macrocell to femtocell in cdma2000-based femtocell systems to emend the performance and enrich user experience with femtocells. In [11] numerical results have been found out by making a co-channel existence for 
proximate indoor femtocells and outdoor macrocell users demonstrating that the desired femtolink provides a robust performance than the macrolink having some equal interference by other femtocells and macrocells. In [12] some simulation results have been shown which interrogates regarding uplink and downlink capacity of macrocell and femtocell demonstrating that the macrocell pursuance derogates due to the locations of femtocell BS and macrocell UE because of femtocell transmit power which is proportional to the number of femtocells. But building these femtocells infrastructure has a huge budget dependency. In [13] a solution to this massive problem has been given by creating FemtoHaul system architecture which efficiently uses relays in the femtocells for bearing the macrocell backhaul traffic and reinforced immense data rates for the cellular subscribers by serving more users with the extant macrocell backhaul capacity. In [14] a proposal has been made on a novel algorithm for creating a neighbour cell list during handover with a minimal but exact number of cells when there are dense femtocells and it is also proposed that CAC effectively handles various calls. In [15] three integrated network architectures have been introduced which has the ability to increase the access capacity by reducing the deployment and operational costs depending on interference management, efficient frequency, xDSL-based backhaul networks quality of service provision, and ingenious handover control issues to apply it in real life scenarios. Interference reduction is one of the main concerns in wireless communication in increasing system performance. These has been discussed in [16] proposing frequency reuse mechanisms which maximizes throughput utilizing different combinations of inner cell radius and allocating frequency depending on the position of the users and the femtocells. Considering random number of cognitive radio, path loss, Raleigh fading aggregate interference using Gamma distribution approximation to perfect closed-form moment generating function with an accurate approximation is derived in [17]. In [18] the research shows a presentation of a mathematical simulation on OFDMA or TDMA based femtocells depending on open and closed access methods according to mobile user density, which suggests OFDMA to be adaptive for the average cellular user connectivity.

\section{B. Summary}

There are different access methods, but among them the open access method has been chosen to find the probability of mobile user connectivity using femtocell which provides robust connectivity than the macrocell users reducing the cost effectiveness of the femtocells. Femtohaul has been used to reduce the traffic of macrocell backhaul.

\section{DIFFERENT TYPES OF ACCESS MECHANISMS OF FEMTOCELLS AND MACROCELLS}

In wireless communication, the users at one end communicate with the person at the other end by building a connection between them. This connection is mainly built by the "Access Points". An access point is mainly a device, with which different wireless devices are connected to a network, e.g., a wireless router. Usually there are built-in routers in most cases in the access points, whereas others must have a connection to a router to serve network access. In each of the two cases the access points are hardwired to network switches or broadband modems. This access control mechanism can be divided into three types:
A. Closed access method.
B. Open access method.
C. Hybrid access method.

\section{A. Closed Access Method}

In closed access method, there will be some authorized and unauthorized subscribers of the femtocells. Only the authorized subscribers would be able to access the femtocells, shown in Fig. 3.

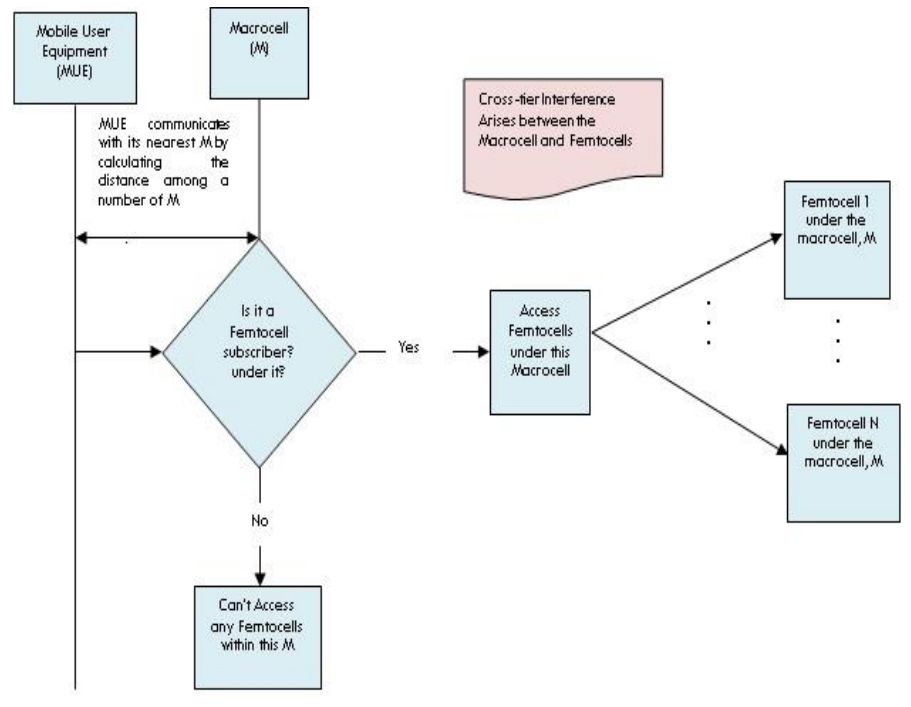

Fig. 3. Flowchart of closed access method.

\section{B. Open Access Method}

In open access method, the subscribers and nonsubscribers of the femtocells would be able to get the service on different condition which is shown in Fig. 4. 


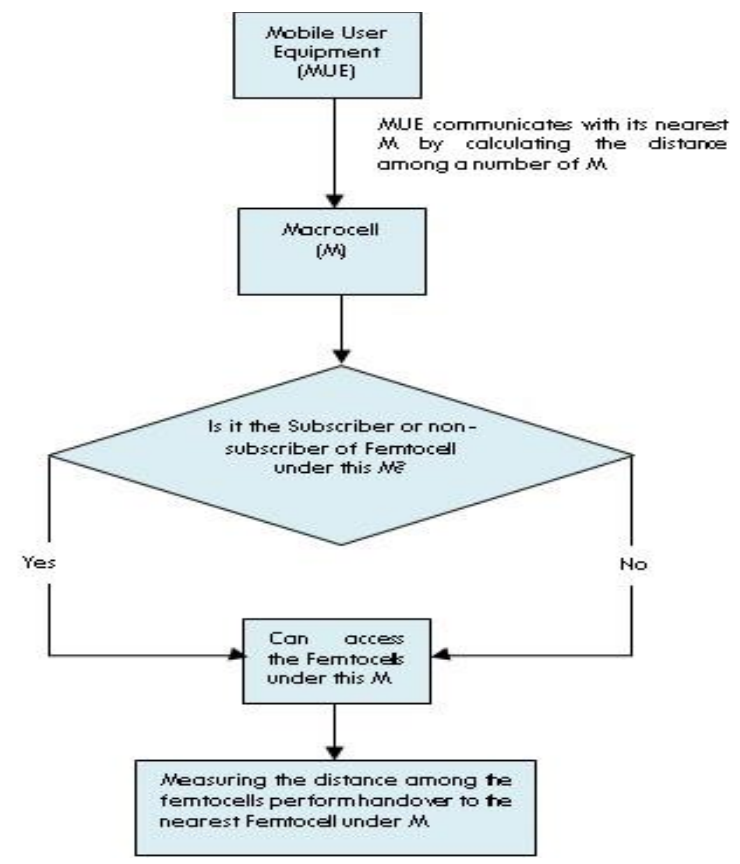

Fig. 4. Flowchart of open access method

\section{Hybrid Access Method}

In hybrid access method, the subscribers and limited number of nonsubscribers of the femtocells would be able to get the service on different conditions, as shown in Fig. 5.

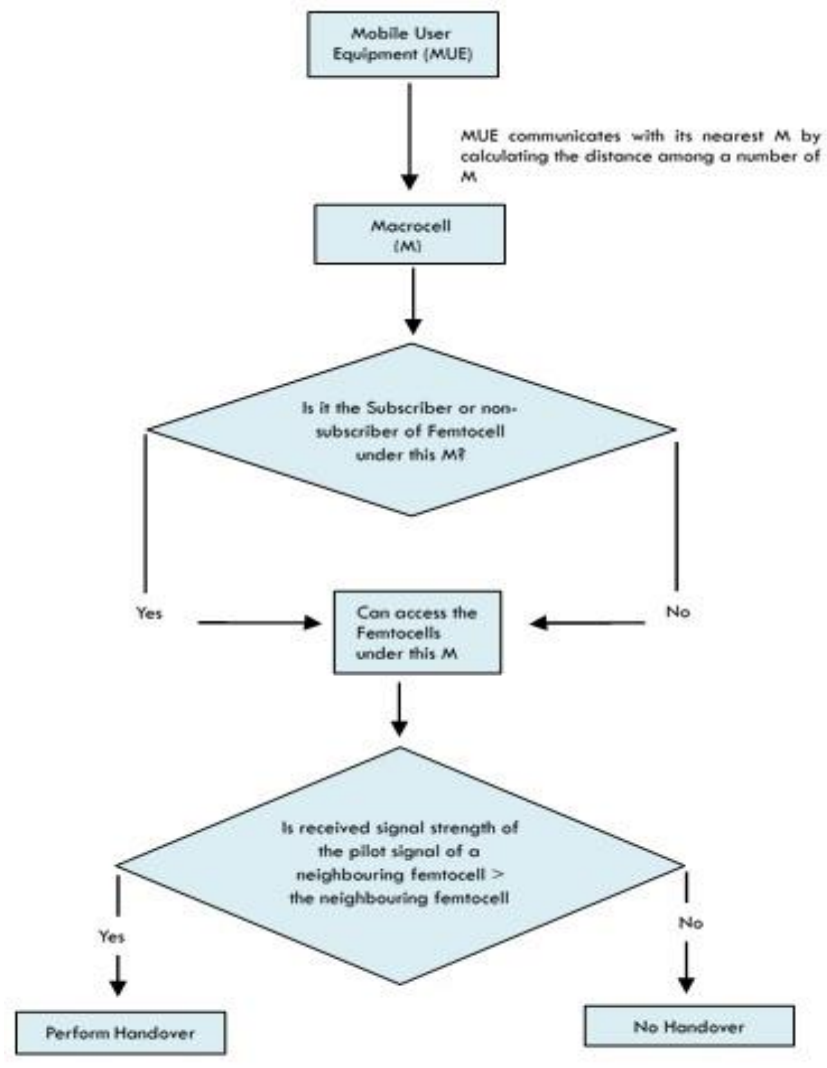

Fig. 5. Flowchart of hybrid access method.

\section{METHODOLOGY}

\section{A. System Model}

The signal to noise plus interference ratio (SINR) of a Femto user at the cell boundary is given by (1):

$$
\operatorname{SINR}=\frac{P_{f} G_{f} R_{f}^{-\alpha}}{N_{0}+I}
$$

Where, $\alpha$ is the path loss exponent

and I, the total interference made by a Femto BS [3] is given by:

$$
I=\sum_{Y_{i} \in \phi_{f}^{P}} G_{Y_{i}} P_{f} d^{-a}
$$

From (1) the advantage of femtocells can be easily realized. For smaller indoor coverages of femtocell, reduced distance between the femtocell and the user leads to higher signal strength. From the Shannon capacity formula,

$$
C=B \log (1+\operatorname{SINR})
$$

The above formula indicates that the increment in signal strength and reduction in interference causes the progression in capacity of the signal. As the femtocell serves only around 2-4 users, it can assign a large portion of these resources (transmit power $P_{f}$ and bandwidth $B$ ) to each subscriber. So, by deploying femtocells, more efficient usage of power and frequency resources can be enabled. As the femtocell is connected to the wired broadband network, the broadband operator provides sufficient QoS over the backhaul. Moreover, the femtocell networks can assist the areas where there is not that much signal coverage by degrading the comparative traffic volume on the macrocell.

Under open access method, a mathematical model for connectivity probability of mobile user in case of communication range as well as mobility factor has been introduced. Let us assume a test femtocell network is deployed within the communication range $(r)$ of a macrocell base station and has allowed open access method in order to enhance the mobile users' connectivity by selecting the closest Femto Access Points. Let us consider, femtocell coverage to be a circle having unit radius. The distance (d) between the centres of femto access point and the macrocell user can be defined by the following equation [6]:

$$
d=1+\beta r
$$

Where, parameter $\boldsymbol{\beta}$ s called the mobility factor of macrocell user that plays an important role to avoid the users from disconnectivity. The area of intersection between a circle of unit radius and circle of radius $r$ as follows [6]:

$$
A(r, \beta)=\left\{\begin{array}{lc}
0, & \beta \geq 1 \\
\alpha(r, \beta), & -1<\beta<1 \\
\pi r^{2}, & \beta \leq-1
\end{array}\right.
$$


Where, [20]

$$
a(r, \beta)=\frac{r^{2}}{\cos \left(\frac{d^{2}+r^{2}-R^{2}}{2 d r}\right)}+\frac{\mathrm{R}^{2}}{\cos \left(d^{2}+r^{2}-R^{2}\right)}-\frac{\sqrt{(-\mathrm{d}+\mathrm{r}+\mathrm{R})}(\mathrm{d}+r-R)(d+r+R)}{2}
$$

Considering $\mathrm{R}=1$.

Let, there are $N$ number of femtocells are overlaid in the macrocell mobile network. So, the probability of macrocell users are not able to connect to any femtocell among $N-1$ is given by [6],

$$
\begin{aligned}
& \alpha(r, \beta)=\left(1-\frac{A(r, \beta)}{\pi r^{2}}\right)^{N-1} \\
& =\left(1-\frac{A(r, \beta)}{\pi \cdot 1^{2}}\right)^{N-1} \\
& =\left(1-\frac{A(r, \beta)}{\pi}\right)^{N-1}
\end{aligned}
$$

So, the probability of connectivity is obtained as [6]

$$
P_{c}=1-\sum_{j=1}^{N}\left(1-\frac{A(r, \beta)}{\pi}\right)^{N-1}
$$

Since a consideration has been made on femtocell network having a scenario of open access method, the femtocells networks are highly and densely populated femtocells. A simplified wireless model neglecting fading has been considered.

Therefore, the probability of a macrocell user which is fully connected in femtocells [6]:

$$
P_{c}=\frac{D_{f}}{D_{u}}\left[1-e^{\left(1-e^{-D_{f} \pi r^{2}}\right) \frac{D_{u}}{D_{f}}}\right]
$$

Where, $D_{f}$ is the density of femto access point and $D_{u}$ is the density of mobile users.

The outage probability of a user under SINR constraint will be [6],

$$
P\left(\operatorname{SINR} \leq \Gamma_{t h}\right)
$$

The above equation can be rewritten according to the active femto access points [6].

$$
1-P\left(\operatorname{SINR} \geq \Gamma_{\text {th }}\right)=1-\frac{D_{f}\left[1-e^{-\left(D_{\text {factive }} \gamma^{\frac{2}{\alpha}}+D_{f}\right) \pi r^{2}}\right]}{\left(D_{f, a c t i v e} \gamma^{\frac{2}{\alpha}}+D_{f}\right)\left[1-e^{\left(-D_{f} \pi r^{2}\right)}\right]}
$$

Where, $D_{f, \text { active }}=P_{c} * D_{u}$

Since channel model is distance dependent. So, considering path loss exponent, the probability of SINR greater or equal to the threshold $\operatorname{SINR} \Gamma_{t h}[1]$,

$$
\begin{aligned}
& P\left(\operatorname{SINR} \geq \Gamma_{\mathrm{th}}\right)= P\left(\frac{P_{f} G_{f} R_{f}^{-\alpha}}{N_{0}+I} \geq \Gamma_{\mathrm{th}}\right) \\
&=P\left\{G_{f} \geq \frac{\Gamma_{\mathrm{th}}\left(N_{0}+I\right)}{P_{f} R_{f}^{-\alpha}}\right\} \\
&=e^{-\Gamma_{\mathrm{th}} N_{0} / P_{f} R_{f}^{-\alpha}} \cdot M_{I}\left(\frac{\Gamma_{\mathrm{th}} I}{P_{f} R_{f}^{-\alpha}}\right)
\end{aligned}
$$

Where,

$$
M_{I}(s)=\exp \left[-2 \pi \lambda \int_{0}^{\infty} \frac{u d u}{1+\frac{u^{\alpha}}{s P_{f}}}\right][17]
$$

So, the outage probability is

$$
1-P\left(\operatorname{SINR} \geq \Gamma_{t h}\right)=1-e^{\Gamma_{t h} N_{o} / p_{f} R_{f}^{-\alpha}} \cdot M_{I}\left(\frac{\Gamma_{t h} I}{P_{f} R_{f}^{-\alpha}}\right)
$$

An analytical work has been done to solve interference by the system models named as interference model, channel models using per tier outage probability considering its coverage. A spectrum division has been used by all proposed solutions. A specific section of the available spectrum needs to be reserved for the femtocells and the rest of them for the macrocells. By avoiding the interference problems, it provides an optimum solution. In this case, among the competing entities of the shared spectrum, one of the femtocells makes a random selection of the reserved spectrum to use a small 
portion. Though the cross-tier interference is reduced potentially, femtocells interference still exists. Moreover, since the femtocells are smaller indoor coverage with 2 to 4 users for each femtocell, the subcarriers reserved for the femtocells remain idle most of the time. This is because users consume their data most of the time either at home (indoor) or at work (outdoor) or rest of the time may be at other places (outdoor). So, this solution causes waste of bandwidth and reduces the spectral efficiency. On the other hand, because of its expensiveness it creates disinterest. Even though, frequency bands sharing is technically more challenging, it is also more appropriate between femtocells and macrocells. The fractional re-use scheme that has been proposed may give a potential solution which is shown in Fig. 6 [19].
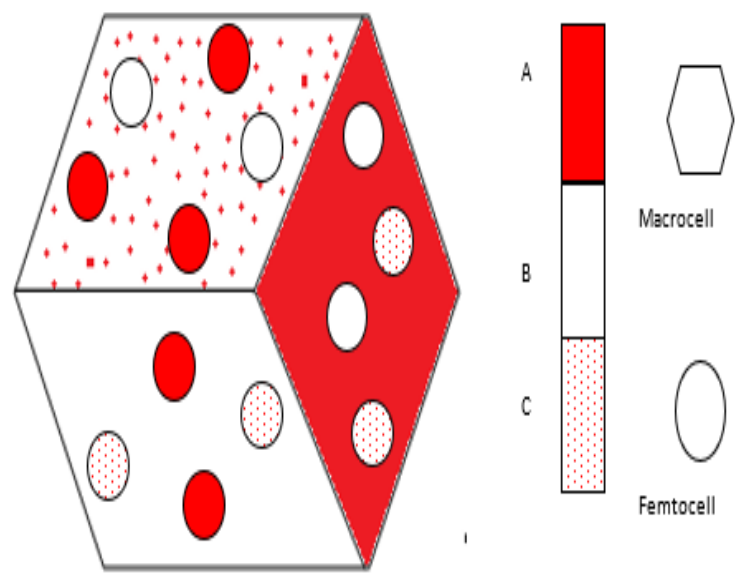

Fig. 6. A probable frequency allocation among femtocells in order to degrade interference.

The above proposed scheme could be used where the frequency re-use factor is static through macro cellular and femto cellular networks. In this case, fractional frequency reuse should be managed very carefully. Because a large number of femtocells might exist inside the macrocells, if any inappropriate frequency allocation among the femtocells has been made it might cause symbolic co-tier interference. Here a model for a small number of femto access points has been proposed giving a solution to co-tier interference and crosstier interference.

In the proposed re-use scheme, at first the frequency band is segregated into three sub-bands $\mathrm{A}, \mathrm{B}$ and $\mathrm{C}$. Then, each macrocell is divided into three portions as sub-bands with respect to center and each of the sub-bands is apportioned to one portion of macrocell. The femtocell allocated in one portion of a macrocell uses one of the other two sub-bands in order to minimize the possibilities of co-tier interference. There is a suggestion for this type of fractional frequency reuse scheme that it can be utilized in SON-based femtocell architectonics. Because, it may reduce the interference by perceptive power optimization and frequency allocation.
When a femtocell is installed, the frequency allocations among the neighbouring femto access points (FAP) will autoreconfigure to get different frequencies used by near femto access points in order to reduce the co-tier interference among the femtocells.

\section{RESULTS AND DISCUSSION}

In this paper, the connectivity and outage probability of mobile user under femtocell/macrocell networks has been derived. A performance measurement model setting different input parameters according to the real cellular networks has been proposed. Here, MathCAD has been used to obtain the numerical solution and graphical representations. The user connectivity probability given by (6) is the function of mobility factor $(\beta)$, communication range $(r)$ and number of femtocells $(N)$. The proposed model is analyzed under three different number of femtocells $N=2, N=5$ and $N=100$.

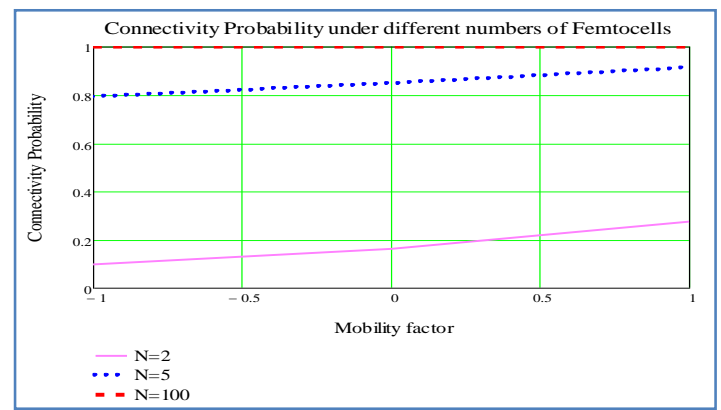

Fig. 7. Variation of connectivity probability against mobility factor.

Fig. 7 shows the probability of mobile user connectivity under different number of femtocells. Here, the mobility factor $(\beta)$ has been considered first which indicates the movement of femto users. The mobility factor $(\beta)$ within the range -1 to 1 has been considered to avoid the users from disconnectedness. This $\beta$ decides whether the user is within the communication range or not. It is clear from the figure that the connectivity probability increases with the increase in communication range especially when the mobility factor $(\beta)$ is varied from 1 towards - 1 . In contrast, it is shown that the connectivity probability decreases when the mobility factor is varied from 1 towards 1. Moreover, it indicates that the system's connectivity performance can be improved with the increase in number of femtocells. This is due to the fact that, when the macrocell is densely populated with femtocells, the probability of mobile users' connectivity increases.

Now, the most important part lies in the relation between connectivity probability and number of mobile users. The connectivity probability is heavily affected by the number of mobile users. Fig. 8 shows that the connectivity probability decreases with increase in the number of mobile users. Moreover, the connectivity probability is low for low density of active femto access points and high for high density of active femto access points which is shown in Fig. 8. The potential results for active femto access points with density, $D f$ $=6$ has been obtained. 


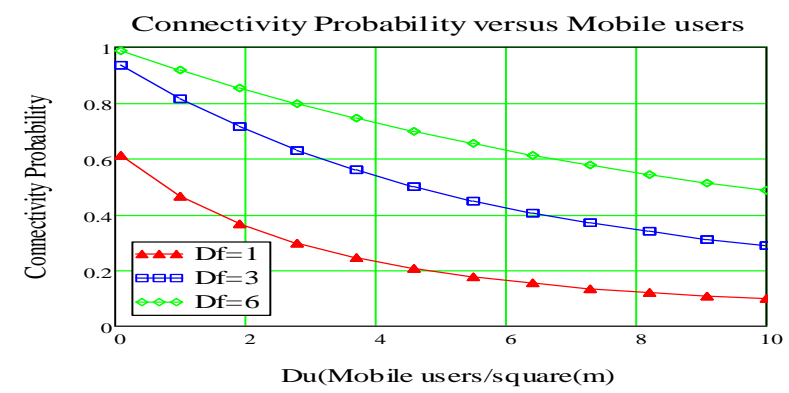

Fig. 8. Variation of connectivity probability against number of mobile.

Finally, Fig. 9 depicts the variation of the outage probability according to the mobile users per square meter under the consideration of the density of femto access points. The outage probability increases with the increase in mobile users and obtained lower value with active femto access points density, $\mathrm{Df}=6$. Therefore, there is a scope of reducing the outage probability by incrementing the connectivity probability beyond this level which is visualized in Fig. 8 and 9 .

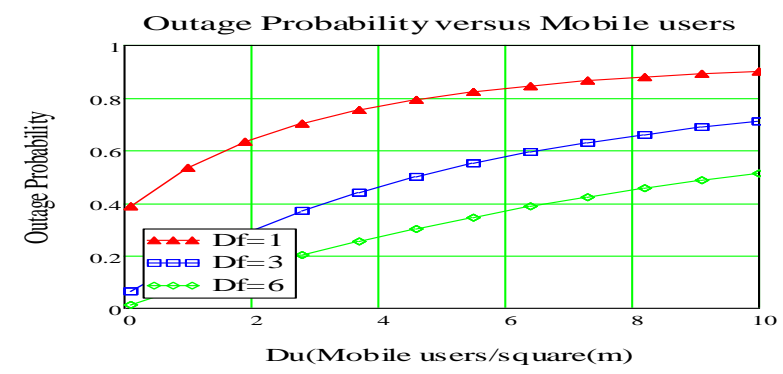

Fig. 9. Variation of outage probability against mobile users.

For Fig. 10, free space path loss exponent $\alpha=2$ and other parameters $R_{f}=15$ and $N_{o}=10^{-12}$ has been considered. The outage probability increases with the increase in density of femto BS and also with the increase in threshold SNR at receiving end.

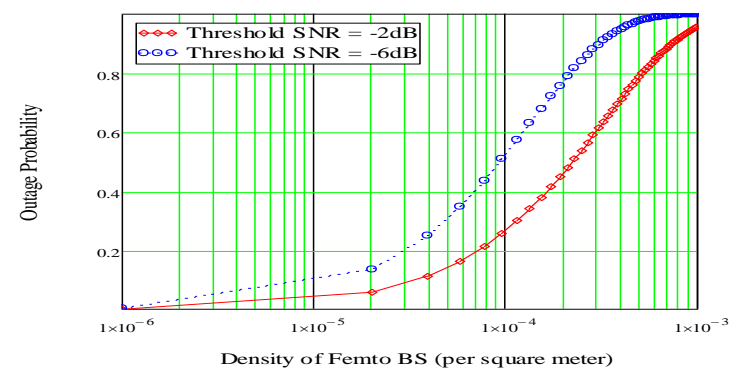

Fig. 10. Variation of outage probability against density of Femto BS taking threshold SINR as a parameter.

The Variation of Outage probability against the threshold of detection under various path loss exponents is shown in Fig. 11. It is clear from the figure that outage probability increases with the increase in threshold of detection or threshold SINR. The impact of path exponent on the outage probability is also cleared from the figure.

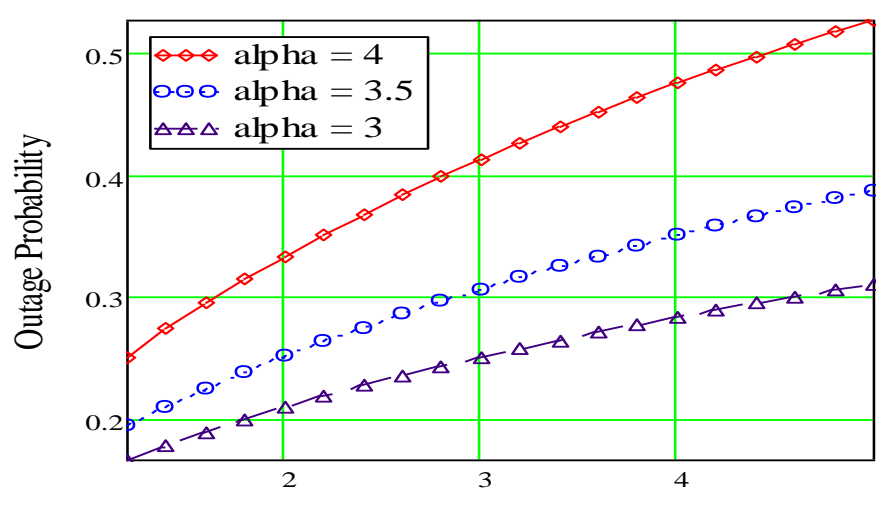

Threshold of Detection

Fig. 11. Variation of outage probability against threshold of detection.

Finally, Fig. 12 shows the variation of Spectral efficiency against SINR. This indicates the maximum achievable spectral frequency through the AWGN channel under SINR.

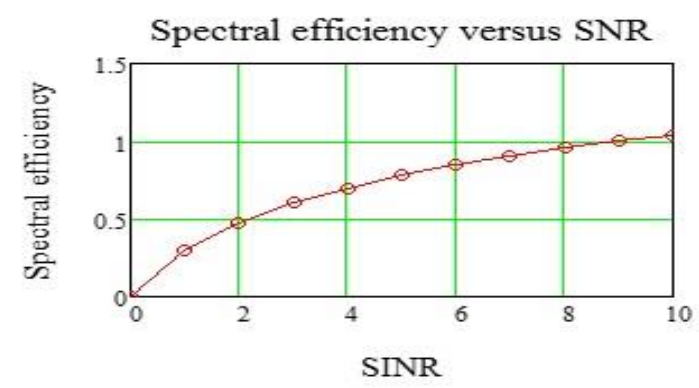

Fig. 12. Variation of spectral efficiency against SINR.

\section{CONCLUSIONS}

Macrocell has a wide area of mobile network connectivity which degrades the performance with the increase in distance of the mobile users from the macrocell access point. This degradation of the connectivity probability problem can be better handled with femtocells under a macrocell. Since Open Access Method (OAM) works with both subscribed and nonsubscribed femtocell users so in this paper a better mobile user connectivity probability has been proposed reducing the outage probability which was tested with different number of mobile users and different SINR threshold and showing those variations of spectral efficiency against Finally, a Fractional Frequency Reuse scheme for SON-based femtocell architecture has been proposed to optimize the interference considering the frequency allocation and power optimization for the mobile users providing improved connectivity probability.

\section{ACKNOWLEDGMENT}

The authors would like to express deep gratitude to their mentors for their impetuous guidance, enthusiastic encouragement and useful rebuke to make this research work. Lastly, the heartiest gratitude goes to their parents for continuous support. 


\section{REFERENCES}

[1] Guillaume de la Roche, Alvaro Valcarce, David L'opez-P'erez, Jie Zhang "Access Control Mechanisms for Femtocells", IEEE Communications Magazine, July 2009.

[2] Shin-Ming Cheng, Weng Chon Ao, and Kwang-Cheng Chen, "Downlink Capacity of Two-tier Cognitive Femto Networks", 21st Annual IEEE international Symposium on Personal, Indoor and Mobile Radio Communications, Print ISBN: 978-1-4244-8017-3 2010, pp. 1301-1306.

[3] Mahsa Derakhshani, Tho Le-Negoc, "Aggregate interference and Capacity-Outage Analysis in a Cognitive Radio Network", IEEE Transactions on Vehicular Technology, JAN 2012, Vol. 61, No. 1, pp. $196-207$.

[4] V. Chandrasekhar and J. G. Andrews, "Femtocell networks: A survey," IEEE Commu. Mag., vol. 46, no. 9, pp. 59-67, Sept. 2008.

[5] S. Yeh, S. Talwar, S. Lee, and H. Kim, "WiMAX femtocells: a perspective on network architecture, capacity, and coverage," IEEE Commu. Mag., vol. 46, no. 10, pp. 58-65, Oct. 2008.

[6] Saied M.Abd El-atty and Z.M. Gharsseldien,"Analytical Model for Mobile User Connectivity in Coexisting Femtocells/Macrocells Networks", International Journal of Wireless \& Mobile Networks (IJWMN) Vol. 4, No. 6, December 2012.

[7] Kanak Raj Chaudhary, DeepeshRawat, EishaMadwal, "Interference Aware \& SINR Estimation in Femtocell Networks", IOSR Journal of Computer Engineering (IOSR-JCE) e-ISSN: 2278-0661, p-ISSN: 22788727 Volume 10, Issue 6 (May. - Jun. 2013), PP 64-69.

[8] Gang Ning, Qinghai Yang, Kyung Sup Kwak, Hanzo, L., "Macro- and Femtocell Interference Mitigation in OFDMA Wireless

[9] Nazmus Saquib, Ekram Hossain, Long Bao Le, and Dong in Kim "Interference Management in OFDMA Femtocell Networks: Issues and Approaches", Wireless Communications, IEEE (Volume:19, Issue: 3), ISSN:1536-128, pp. 86-95, June 2012.

[10] HumbletP.Airvana, Raghothaman, B., Srinivas, A., Balasubramanian, S., Patel, C., Yavuz, M. "System design of CDMA2000 femtocells", Communications Magazine, IEEE (Volume:47, Issue: 9), ISSN: 01636804, pp. 92-100, September 2009.
[11] PekkaPirinen "Co-channel co-existence study of outdoor macrocell and indoor femtocell users", Wireless Conference (EW), 2010 European, ISSN: 978-1-4244-5999-5 pp. $207-213$.

[12] Chang Seup Kim, Bum-Gon Choi, Ju Yong Lee, Tae-Jin Lee, HyunseungChoo, and Min Young Chung "Femtocell Deployment to Minimize Performance Degradation in Mobile WiMAX Systems".

[13] AyaskantRath, Sha Hua and Shivendra S. Panwar "FemtoHaul: Using Femtocells with Relays to Increase Macrocell Backhaul Bandwidth", INFOCOM IEEE Conference on Computer Communications Workshops, 2010, pp. 1-5, March 2010.

[14] Mostafa Zaman Chowdhury and Yeong Min Jang, "Handover management in high-dense femtocellular networks", EURASIP Journal on Wireless Communications and Networking, A Springer Open Journal, Vol. 2013, No. 6, pp. 1-21, 7 January, 2013.

[15] Mostafa Zaman Chowdhury, Yeong Min Jang and Zygmunt J. Haas "Network Evolution and QOS Provisioning for Integrated Femtocell/Macrocell Networks", International Journal of Wireless \& Mobile Networks (IJWMN), Vol.2, No.3, August 2010.

[16] H Bouras, C., Kavourgias, G., Kokkinos, V., Papazois, A., "Interference Management in LTE Femtocell Systems Using an Adaptive Frequency Reuse Scheme", Wireless Telecommunications Symposium (WTS), 2012, Print ISBN: 978-1-4577-0579-3, pp. 1-7.

[17] Sachitha Kusaladharma, Chintha Tellambura, "Aggregate Interference Analysis for Underlay Cognitive Radio Networks",2012, Wireless Communications Letters, IEEE, Volume 1, Issue 6, pp.641 - 644.

[18] Ping Xia, Vikram Chandrasekhar, Jeffrey G. Andrews "Femtocell Access Control in the TDMA/OFDMA Uplink", Global Telecommunications Conference (GLOBECOM 2010), IEEE, ISSN: 1930-529X, pp. 1-5, December 6-10, 2010.

[19] Rizwan Ghaffar, Raymond Knopp "Fractional Frequency Reuse and Interference Suppression for OFDMA Networks", WIOPT 2010, 8th International Symposium on Modeling and Optimization in Mobile, Ad Hoc, and Wireless Networks, Avignon, France, 31 May-4 June 2010.

[20] Wolfram Math World: http://mathworld. wolfram.com/CircleCircleIntersection.html. 